\title{
Advances in large eddy simulation methodology for complex flows
}

\author{
Parviz Moin \\ Center for Turbulence Research, Stanford University and NASA-Ames, USA
}

\begin{abstract}
A review is provided of the recent advances in the derivation of the constitutive equations for large eddy simulation, subgrid scale modeling, wall modeling and applications of LES to turbulent combustion. The majority of the paper focuses on a review of two numerical methods for LES in complex geometry: the immersed boundary method and an unstructured mesh scheme. The latter scheme is applied to LES of a sector of a combustor of an operational gas turbine engine. (C) 2002 Published by Elsevier Science Inc.
\end{abstract}

\section{Introduction}

The advent of massively parallel computers and affordable workstation clusters has stimulated industry interest in applying LES to engineering flows. Resolution of large turbulent eddies is required in many applications such as those involving turbulent mixing and aerodynamic noise. Most of these applications require computation of turbulence in complex geometries. Unfortunately, in most cases, numerical methods used for efficient RANS computations are not appropriate for LES. In contrast to RANS where the steady or unsteady solutions are smooth, turbulent flows have broad band spectra, and most numerical methods used for robust RANS computations are inaccurate in the representation of the medium to small resolved eddies in LES. For example, the use of upwind schemes is prevalent in industrial CFD and it has been demonstrated that the inherent numerical dissipation of even the high order upwind schemes can lead to excessive dissipation of the resolved turbulent structures (Mittal and Moin, 1997). If the purpose of using LES is to capture the turbulence structures, which are not available from RANS, then the numerical methods used in LES should be sufficiently accurate in representing their dynamics, rather than remove them by artificial dissipation.

Application of LES to industrial problems requires good subgrid scale models, fast computers, accurate and robust numerical methods suitable for complex configurations and reliable experimental data for validation.

E-mail address: moin@stanford.edu (P. Moin).
Of these required ingredients, development of numerical methods has received the least attention. Although significant advances have been made in subgrid scale model development, the models await to be tested in truly complex heterogeneous turbulent flows, so that the need for improvements and further research can be identified. Fundamental advances in numerical algorithms are needed before this testing can take place and LES can transition to industry. The focus of this review is on the development of numerical methods for LES.

In the main body of the paper I describe two numerical methods that have been developed at the Center for Turbulence Research for LES in complex domains. Recent major advances in other components of LES are highlighted in this section. One approach is based on the immersed boundary (IB) method where body forces are used to enforce the boundary conditions and hence account for the geometry. In the past typical calculations with the IB method were done on a Cartesian mesh, but recently it has been used effectively in conjunction with curvilinear and unstructured grids. Applications of this method include the flow in an impeller stirred tank, flow around a road vehicle with drag reduction devices and tip clearance flow in a stator/rotor combination. The second numerical method is designed for unstructured grids with arbitrary elements. This is a fully conservative method and is being used for computations in the combustor of a gas turbine jet engine.

\subsection{Filtering and constitutive equations}

With the development of spatial filters that commute with differentiation, the governing LES equations are 
now rigorously derived in complex domains (Vasilyev et al., 1998; Marsden et al., 2002). It is desirable for the LES filter width to be uncoupled from the computational grid. That is, grid refinement while fixing the filter width should lead to the solution of the LES equations instead of the DNS solution. Recent LES studies of channel flow with three-dimensional spatial filters have reaffirmed the conclusions of Piomelli et al. (1988) regarding consistency of the subgrid scale model and the filter. For example, a filter that removes energy from a broad range of scales produces better results when used in conjunction with a scale similarity model instead of the Smagorinsky's model, and a nearly sharp cut-off filter when used in conjunction with the Smagorinsky model leads to results in good agreement with the DNS data (Gullbrand, 2001).

\subsection{Subgrid scale modeling}

Over the past decade several advances have been made in subgrid scale modeling which are particularly appropriate for LES in complex geometries. Complex flows usually contain multiple flow regimes (boundary layers, wall jets, wakes, etc.) and it has been demonstrated that models with a fixed coefficient require tuning of their coefficients in each flow regime. The dynamic modeling approach (Germano et al., 1991; Moin et al., 1991; Ghosal et al., 1995) does not suffer from this limitation because the model coefficient is a function of space and time, and is computed rather than prescribed. In addition, it has the proper limiting behavior near walls without ad hoc damping functions and does behave appropriately in the transition regions. These are all very important features for LES in complex domains.

Other significant developments in the subgrid scale modeling area are Domaradzki's subgrid scale estimation model, the deconvolution model of Stolz et al. (2001), and the multi-scale formulation of Hughes et al. (2001). Domaradzki and Loh (1999) use extrapolation from the resolved scales to subgrid scales to construct the subgrid scale velocity fluctuations and stresses. The model has an adjustable parameter which should be possible to compute dynamically. Stolz et al's approach is an algorithmic procedure as opposed to phenomenological modeling; it uses regularized deconvolution of the velocity field to estimate the unfiltered flow field. Hughes et al. have shown that better results are obtained if the governing equations for LES are split into the large and small scale equations, and the eddy viscosity model is only applied to the small scale equations. Although, this approach is trivial to implement in the Fourier space and has produced excellent results, extension to complex geometry appears to be straight forward with a variational formulation as proposed by Hughes et al. (2001).

\subsection{Wall modeling for LES}

One of the pacing items for application of LES to high Reynolds number boundary layers is the treatment of the wall layer structures. The subgrid scale models are not designed to account for the highly deterministic near wall structures. Therefore, a practical approach for the treatment of the wall layer has been to model it all together. Nicoud et al. (2001), using optimal control theory, have shown that such models should account for subgrid scale modeling as well as numerical errors. To account for these errors the control algorithm significantly energized the wall layer so that the simulations could predict the logarithmic mean velocity profile at very high Reynolds numbers and with coarse resolutions.

Wang and Moin (2001) use the RANS approach in the near wall region (Balaras et al., 1996), but incorporate a new dynamic approach to adjust the model coefficient. The basic rationale for the adjustment is that when a RANS type eddy viscosity is used in the wall layer equations, which includes non-linear convective terms, its value must be reduced so that the eddy viscosity would account for only the unresolved part of the Reynolds shear stress. From the unsteady boundary layer equations one can derive the following expression for the mean wall shear stress:

$$
\begin{aligned}
\bar{\tau}_{w 1}=\mu & \left.\frac{\partial U_{1}}{\partial x_{2}}\right|_{x_{2}=0} \frac{\rho}{\int_{0}^{\delta} \frac{\mathrm{d} y}{v+v_{t}}}\left\{U_{\delta_{1}}-\frac{1}{\rho} \frac{\partial P}{\partial x_{1}} \int_{0}^{\delta} \frac{y \mathrm{~d} y}{v+v_{t}}\right. \\
& \left.-\int_{0}^{\delta} \frac{\int_{0}^{y} \frac{\partial}{\partial x_{j}} \overline{u_{1} u_{j}} \mathrm{~d} y^{\prime}}{v+v_{t}} \mathrm{~d} y\right\}
\end{aligned}
$$

The eddy viscosity, $v_{t}$ was a mixing length model with constant of proportionality equal to the von Karman constant, $\kappa$. The pressure gradient is imposed as a constant in the wall normal direction and is obtained from the outer LES. If the standard value of $\kappa=0.4$ is used, the predicted wall shear stress would be too large. Wang and Moin adjust $\kappa$ dynamically to match the LES and RANS eddy viscosities at the matching points. This approach has produced results in good agreement with experiments and wall resolved LES of separated flow over an asymmetric trailing-edge of a hydrofoil at $10 \%$ of the cost of a wall-resolved LES. In Fig. 1 the dynamic $\kappa$ is plotted near the trailing edge of a hydrofoil of thickness $h \cdot x_{1}=0$ corresponds to the trailing edge. It can be seen that the matched $\kappa$ is significantly below the value of 0.4 .

\subsection{LES of turbulent combustion}

The most recent and promising application of LES has been in turbulent combustion (Vervisch and Poinsot, 1998). This is an area where the fundamental 


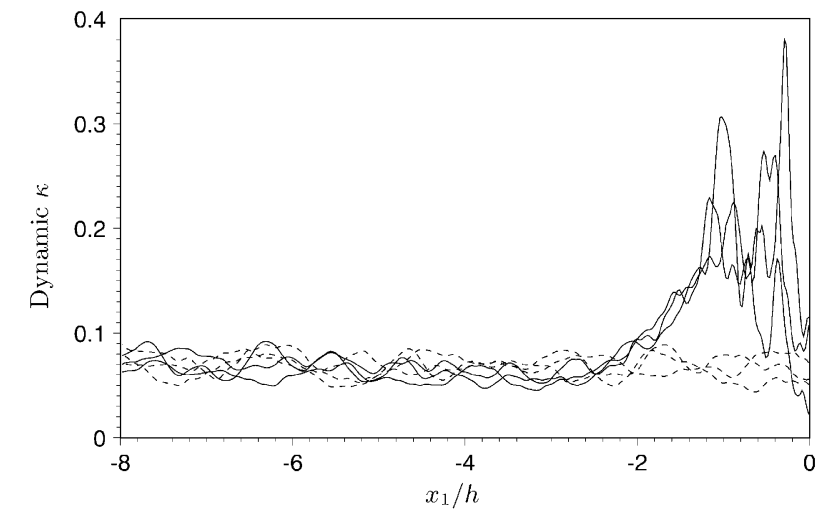

Fig. 1. Dynamic $\kappa$ for the mixing-length eddy viscosity model at three time instants. (-) suction side; (---) pressure side.

advantages of LES (over RANS) has been called into question because chemical reactions take place at very small scales. However, recent developments and results have proven otherwise. In particular, the so called indirect approaches which use the mixture fraction as the fundamental variable have proven to be both economical and accurate for prediction of diffusion flames. In the flamelet approach the chemistry (often complex mechanisms) is pre-computed in look-up tables which provide the chemical variables such as species concentrations and temperature as a function of the quantities readily available from LES. The mixture fraction and additional tracking parameters serve as pointers to the table.

One of the challenges facing computation of turbulent combustion has been the prediction of highly unsteady lifted flames which occur in gas turbine combustors. Approaches based on fast chemistry and steady flamelets predict attached flames leading to inaccurate turbulence and temperature statistics in the combustor. The first LES prediction of a lifted flame was recently completed by Pierce and Moin (2001). To account for unsteady effects they introduced a reacting scalar which tracks the progress of the reaction. The progress variable acts as a new pointer to the flamelet library. The computations are in very good agreement with the experimental data in the profiles of velocity, mixture fraction, primary species concentrations and their fluctuations. However, prediction of the so called secondary variables that constitute pollutants such as $\mathrm{CO}$ and $\mathrm{NO}_{x}$ are not in satisfactory agreement with the data. Pitsch and Steiner (2000) have successfully predicted pollutant concentrations in a piloted methane-air diffusion flame using an unsteady flamelet model formulation. Pitsch is now applying the same method to the prediction of the lifted flame as in Pierce and Moin. He has also shown that accounting for the fluctuations in the dissipation of mixture fraction (which can only be obtained from LES and not from RANS) was a key in the success of these computations.

\section{LES with the immersed boundary technique}

The IB technique allows the computation of flow around complex objects without requiring the grid lines to be aligned with the body surface. The governing equations are solved on an underlying grid (in principle it can be structured or unstructured) which covers the entire computational domain without the bodies; no-slip boundary conditions are enforced via source terms (body forces) in the equations (Verzicco et al., 2000b).

A boundary body-force term $\mathbf{f}$ is added to the incompressible equations to yield,

$$
\begin{aligned}
& \frac{\mathrm{D} \overline{\mathbf{u}}}{\mathrm{D} t}=-\rho^{-1} \nabla \bar{P}+\nabla \cdot\left\{\tilde{v}\left[\nabla \overline{\mathbf{u}}+(\nabla \overline{\mathbf{u}})^{\mathrm{T}}\right]\right\}+\mathbf{f} \\
& \nabla \cdot \overline{\mathbf{u}}=0
\end{aligned}
$$

The effective viscosity $\tilde{v}$ is the sum of the molecular viscosity and the subgrid-scale viscosity, $v_{t}$ which is determined using the dynamic procedure.

The time-discretized version of Eq. (2.1) can be written as,

$\overline{\mathbf{u}}^{n+1}-\overline{\mathbf{u}}^{n}=\Delta t(\mathrm{RHS}+\mathbf{f})$

where $\Delta t$ is the computational time step, RHS contains the non-linear, pressure, and viscous terms, and the superscript denotes the time-step level.

In order to impose $\overline{\mathbf{u}}^{n+1}=\overline{\mathbf{v}}_{\mathrm{b}}$, on the body, the forcing f must be,

$\mathbf{f}=-\mathrm{RHS}+\frac{\overline{\mathbf{v}}_{\mathrm{b}}-\overline{\mathbf{u}}^{n}}{\Delta t}$

in the region where we wish to mimic the solid body, and zero elsewhere (Fadlun et al., 2000). In general, the surface of the region where $\overline{\mathbf{u}}^{n+1}=\overline{\mathbf{v}}_{\mathrm{b}}$ does not coincide with a coordinate line. The value of $\mathbf{f}$ at the node closest to the surface, but outside the solid body, is linearly interpolated between the value that yields $\overline{\mathbf{v}}_{\mathrm{b}}$ on the solid body and zero in the interior of the flow domain. This interpolation procedure is consistent with a centered second-order finite-difference approximation, and the overall accuracy of the scheme remains second-order.

To facilitate the application of the IB method to complex configurations a geometry pre-processor has been developed by G. Iaccarino at CTR. The immersed objects are described using stereo-lithography (STL) format; the STL representation of a surface is a collection of unconnected triangles of sizes inversely proportional to the local curvature of the original surface. The geometrical preprocessor uses the STL surface description and the three-dimensional underlying grid to generate all the interpolation data required to enforce the boundary conditions in the IB flow solver. As a first step the geometrical module performs the separation (tagging) of the computational cells into dead (inside the body), alive (outside the body) and interface (partially inside). This step is based on a ray tracing procedure 


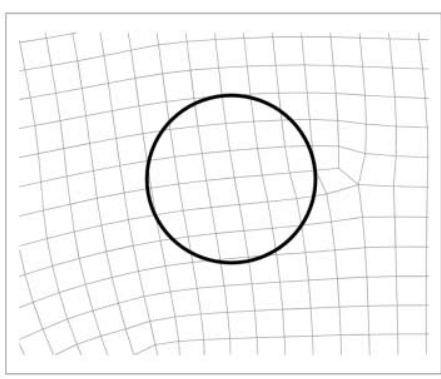

(a)

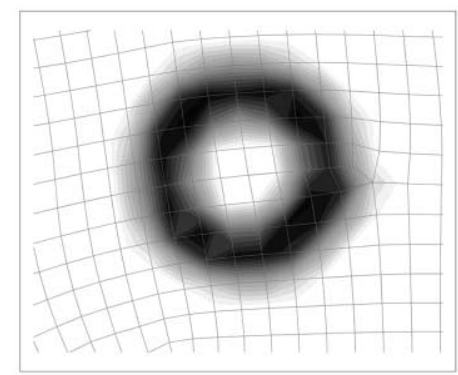

(c)

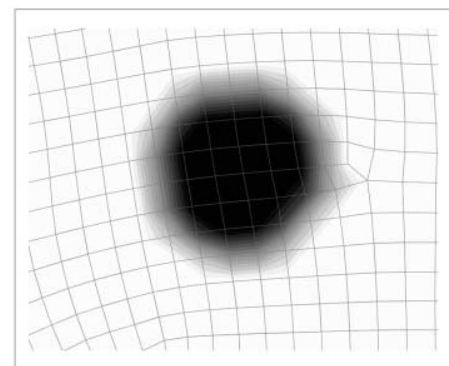

(b)

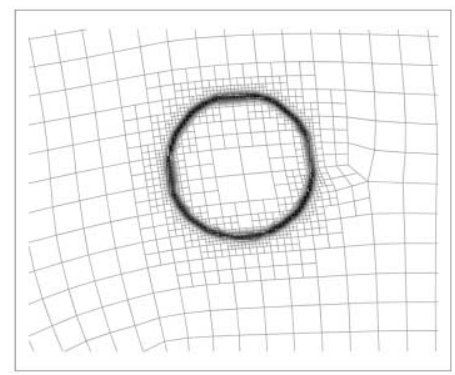

(d)

Fig. 2. Grid refinement procedure: (a) IB and initial unstructured grid; (b) tagging function; (c) gradient of the tagging function; (d) refined grid.

(O'Rourke, 1998) used in computer graphics to render and shade three dimensional objects.

An automatic grid-refinement procedure has been developed to improve the representation of the body on the underlying grid. In Fig. 2 a circular boundary is immersed on an underlying unstructured grid (Fig. 2a). The tagging function $T$ is shown in Fig. $2 \mathrm{~b}$ for an initial coarse mesh; the dark area corresponds to internal cells $(T=-1)$ whereas the white area corresponds to fluid cells $(T=1)$. The numerical gradient of this function is shown in Fig. 2c and its value is proportional to the local grid size. By successively halving the cells until this gradient exceeds a prescribed value the grid and the corresponding sharper geometrical representation in Fig. 2d is obtained.

To be able to use the geometry pre-processor, mesh adaptation capability must be available in the basic underlying CFD code. Presently, most LES codes do not have such a capability. The following examples demonstrate applications of the IB method in existing structured LES codes written in cylindrical, Cartesian and curvilinear coordinates without zonal or mesh adaptation capability.

\subsection{LES of a stirred tank mixer}

As an example of the IB method with an underlying mesh in cylindrical coordinates, the LES/IB solver has been used to investigate the flow in a cylindrical unbaffled tank stirred by an impeller located at mid-height of the tank, rotating at constant velocity $\Omega$ (Verzicco

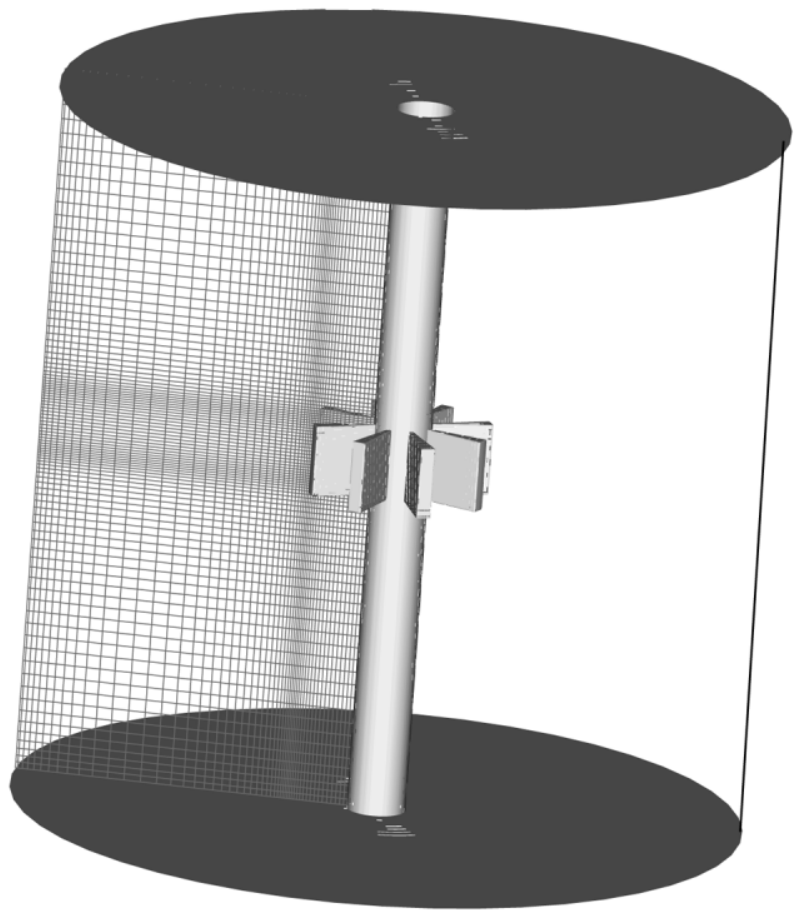

Fig. 3. Tank configuration and computational grid in a meridional plane (only one out of every six grid-points are shown).

et al., 2000a). The impeller has eight blades equally spaced over the azimuthal span (Fig. 3). A computational grid made up of $192 \times 102 \times 97$ nodes (in the vertical, radial and azimuthal directions respectively) has been used. The grid is uniform in the azimuthal 
direction; a section of it is shown in Fig. 3. No slip boundary conditions are imposed on the impeller, the shaft, the bottom and external surfaces of the tank; a slip boundary condition is imposed on the upper boundary of the computational domain. The Reynolds number based on the rotational speed and the blade radius $\left(R_{\mathrm{b}}\right)$ is $R e=1636$ (Dong et al., 1994).

Flow features are presented in Fig. 4 in terms of azimuthally averaged velocity vectors, instantaneous velocity magnitude and turbulent kinetic energy. Quantitative comparisons between the simulations and experimental data is reported in Fig. 5 in terms of radial profiles of azimuthal, radial and vertical velocity components. The present simulations are in very good agreement with the measurements; in particular the peaks of the azimuthal and radial velocity close to the impeller are very well captured. Reynolds-averaged Navier-Stokes simulations with the $\kappa-\epsilon$ model were also carried out for the same configuration (Verzicco et al., 2000a) and show some disagreement with the measurements, especially for the radial velocity which is strongly overpredicted.

The Reynolds number in the configuration considered is low enough to make LES competitive with RANS simulations in terms of computational cost; the disagreement of the RANS predictions with the measured data is due to the presence of large scale unsteadiness and heterogeneous flow (laminar/turbulent). The dynamic model is ideal in this case because of its adaptability to different flow regimes.

\subsection{LES of a road-vehicle with drag reduction devices}

The Cartesian IB technique has been used to simulate the flow around a square-back road-vehicle with drag reduction appendices attached to its base (Verzicco et al., 2001). The road vehicle is mounted on a bi-convex airfoil which is mounted on the floor. The objective is to study the unsteady dynamics of the wake and the modifications induced by the drag reduction devices; experimental data are available for comparison (Khalighi et al., 2001). The baseline configuration is shown in Fig. 6; the simulations are performed on a Cartesian grid consisting of $220 \times 140 \times 257$ points in the streamwise, vertical and spanwise directions respectively. The experimental Reynolds number based on the free-stream velocity and the model height $(H)$ is $R e=170,000$.

Time-averaged results are shown in Fig. 7 for the three configurations analyzed at $R e=20,000$. The flow patterns in the near-wake recirculation region are very different; the results for the baseline square-back configuration show a strong interaction between the base recirculation and the boundary layer on the bottom wall. The ground separation disappears at $R e=100,000$ in accordance with the experiments.

In Fig. 8 time-averaged streamwise velocity profiles are shown at two sections downstream of the base for the square-back configuration. The measurements are compared with two LES simulations performed at $R e=20,000$ and 100,000; the high Reynolds number simulations are in better agreement with the experiment. The defect velocity as well as the length of the recirculation region are accurately captured. The low Reynolds number simulations agree qualitatively with the measurements but strongly overpredict the thickness of the bottom-wall boundary layer. The high Reynolds number results have also been compared to the experiments in terms of drag coefficients: values of 0.291 for the square back and 0.223 for the boattail were computed

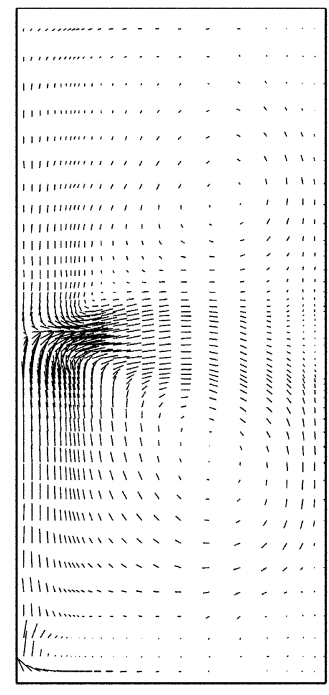

(a)

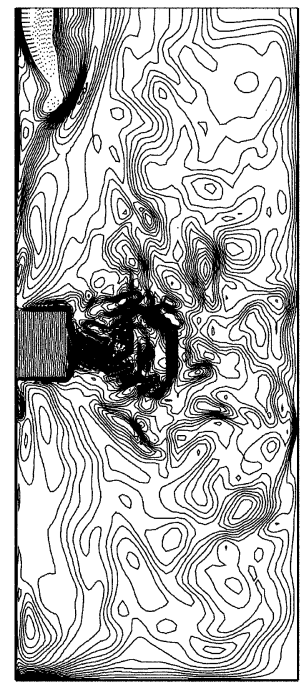

(b)

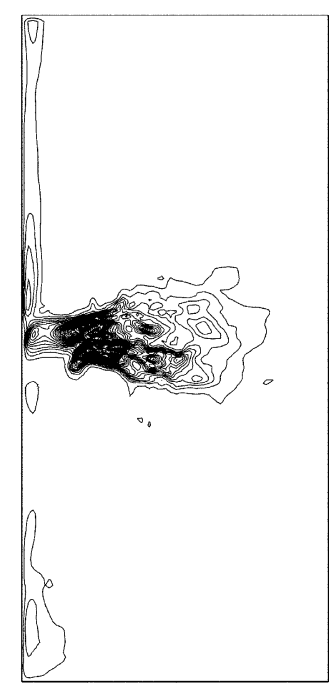

(c)

Fig. 4. Contour plots of azimuthally averaged velocity vectors (a), instantaneous velocity magnitude (b), and turbulent kinetic energy (c) in a meridional plane crossing a blade. 

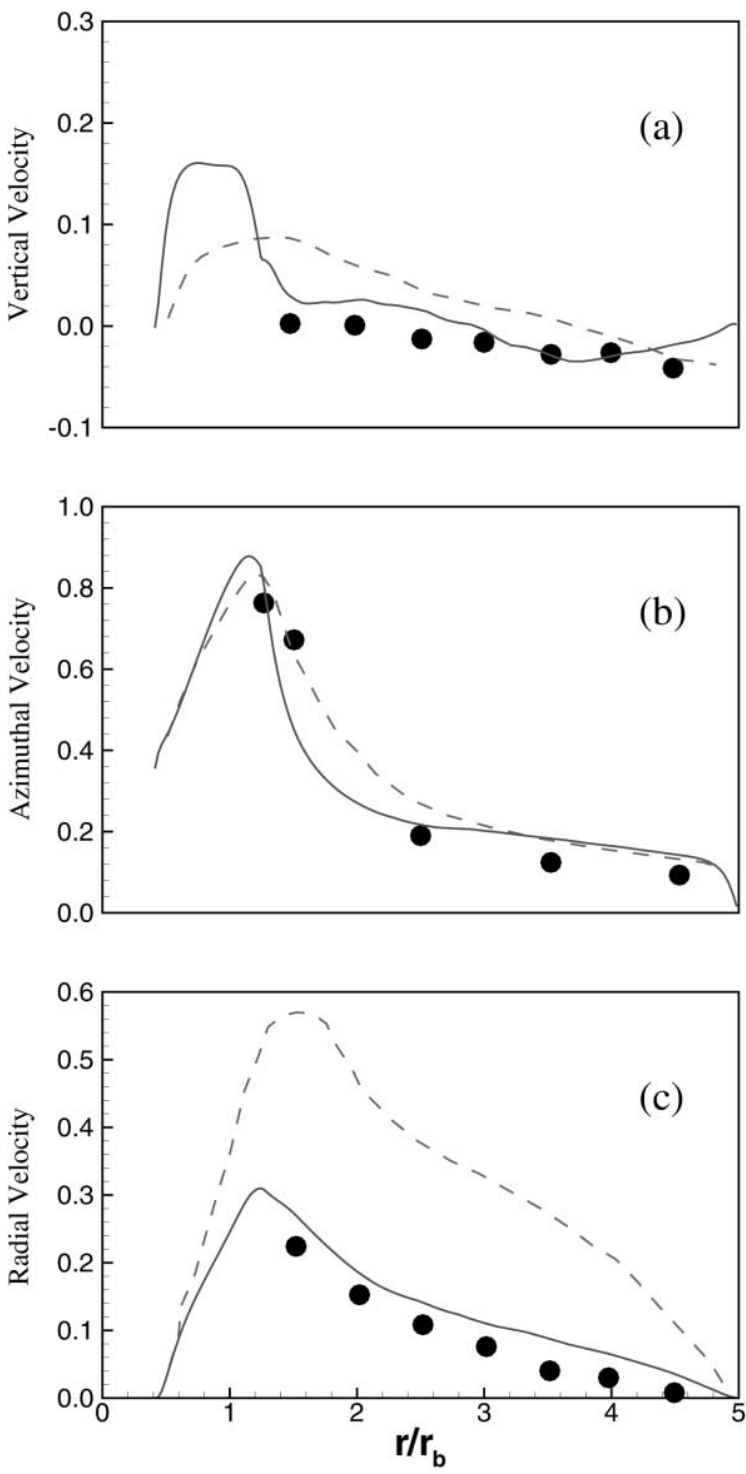

Fig. 5. Radial profiles of averaged azimuthal velocity components in the middle of the tank. Symbols: experiments (Dong et al., 1994), (-) present LES; (----) RANS simulations (Verzicco et al., 2000a).

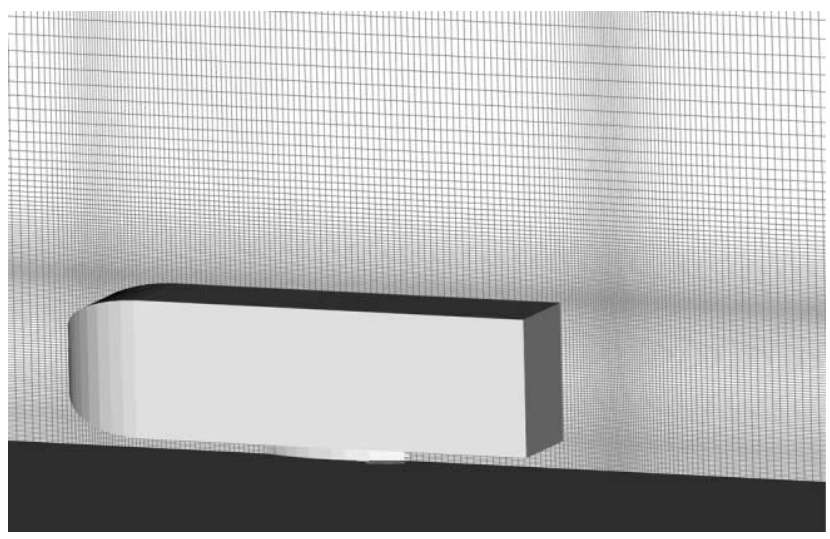

Fig. 6. Road-vehicle configuration and the computational grid in the symmetry plane (only one out of every four grid-points are shown).

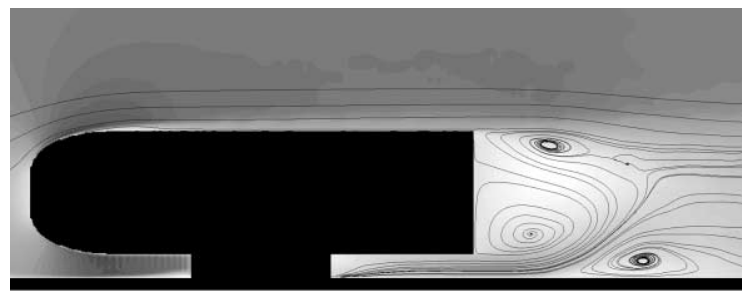

a)

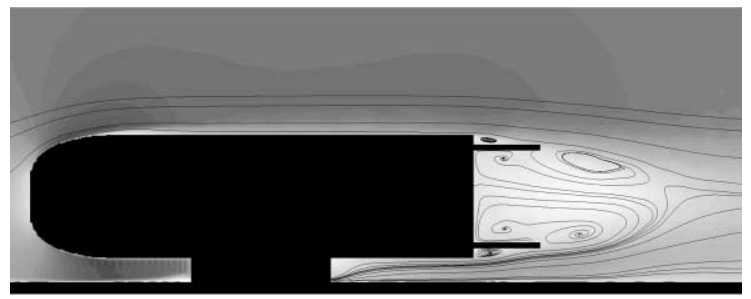

b)

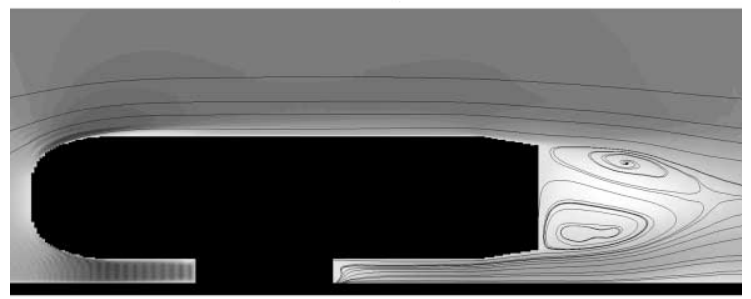

c)

Fig. 7. Flow patterns in the symmetry plane superimposed on contours of time-averaged streamwise velocity. $R e=20,000$ (a) baseline squareback geometry; (b) square-back with base plates, (c) boat-tail base.

from the LES simulations, as compared to 0.3 and 0.23 respectively from the measurements.

This example demonstrates the utility of the IB method in the design process, where the effect of small geometrical changes on the overall performance is desired. The use of a simple Cartesian mesh allows performing the simulations very efficiently without the need to re-generate computational grids for every configuration.

\subsection{Immersed boundary method in curvilinear coordinates}

A severe challenge to the IB method for computing high Reynolds number flows is the near-wall resolution. While mesh embedding, as discussed in the Section 1.3, or the use of a wall model offer significant relief, the resolution requirement can be most efficiently addressed through grid clustering in the wall normal direction if one set of grid lines is parallel or nearly parallel to the boundary. Hence, on a Cartesian mesh, the IB method works best when the bounding surfaces are nearly flat (as in the previous example) and perpendicular to one another, or if the object is slender.

At moderate to high Reynolds numbers and in the presence of complex boundary shapes, it is often 

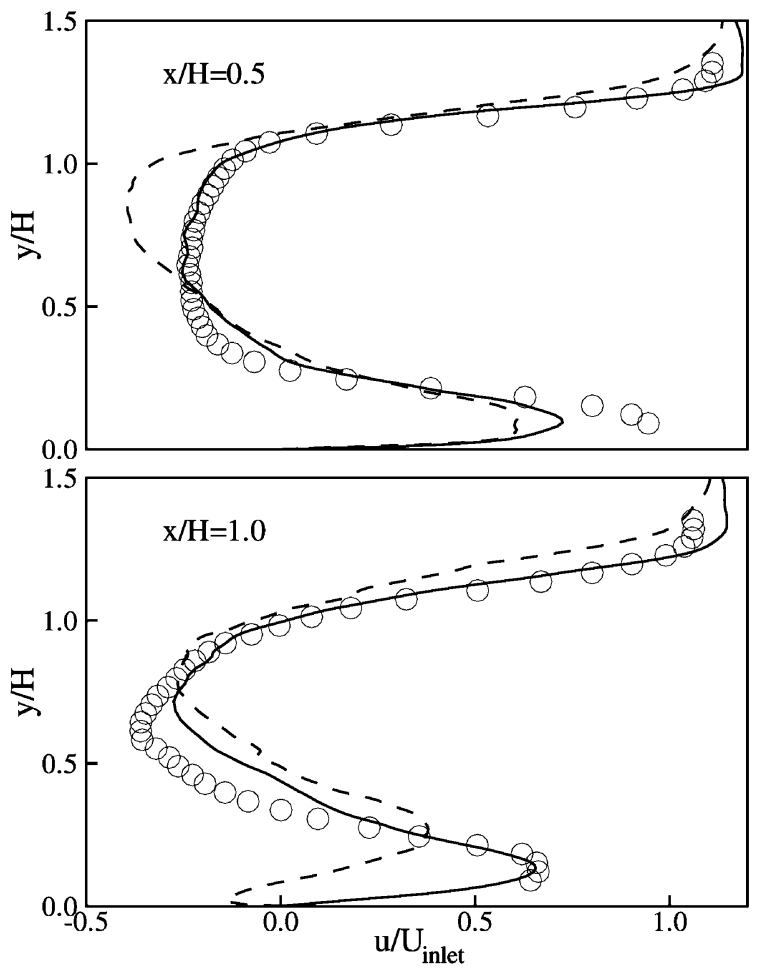

Fig. 8. Streamwise velocity profiles in the wake of the square-back configuration. Symbols: experiments (Khalighi et al., 2001); (- - - ) LES at $R e=20,000 ;(-)$ LES at $R e=100,000$.

advantageous to combine the IB technique with a structured curvilinear mesh topology. This novel approach is applied in an ongoing large-eddy simulation of the tip-clearance flow in a stator-rotor combination (You et al., 2002). A schematic of the flow configuration is shown in Fig. 9 (Wang, 2000). The chord Reynolds number is of $\mathrm{O}\left(10^{5}\right)$. The rotor stage simulation is carried out in a frame of reference attached to the rotor, with the endwall moving at a velocity equal and opposite to the rotor velocity. The tip-gap region between the rotor tip and the endwall presents considerable grid topology and resolution challenges. It has been a major obstacle to the accurate prediction of this flow.

A commonly used mesh topology for the tip clearance flow is the body-fitted $H$-type mesh. This mesh topology is often extended to the "embedded $H$-type mesh" to facilitate the treatment of the tip-clearance region (e.g., Kunz et al., 1993). However, the embedded $H$-mesh has significant drawbacks. As shown in Fig. 10, the blade surface in an $x-y$ plane (see Fig. 9 for coordinate definition) is mostly represented by longitudinal grid lines except near the leading and trailing edges, where it is represented by the transverse grid lines. The number of longitudinal grid lines inside the airfoil is determined by the resolution requirements in the tipgap. This causes convergence of the longitudinal grid lines in the leading and trailing edge regions, leading to high aspect and stretching ratios, which can cause dif-

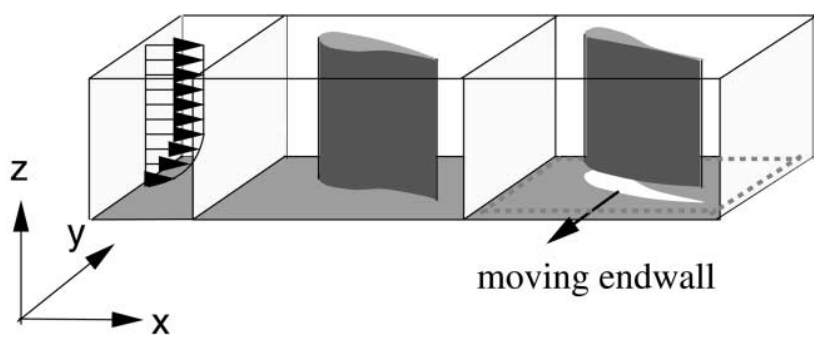

Fig. 9. Schematic of flow configuration for LES of tip-clearance flow in a stator-rotor combination.

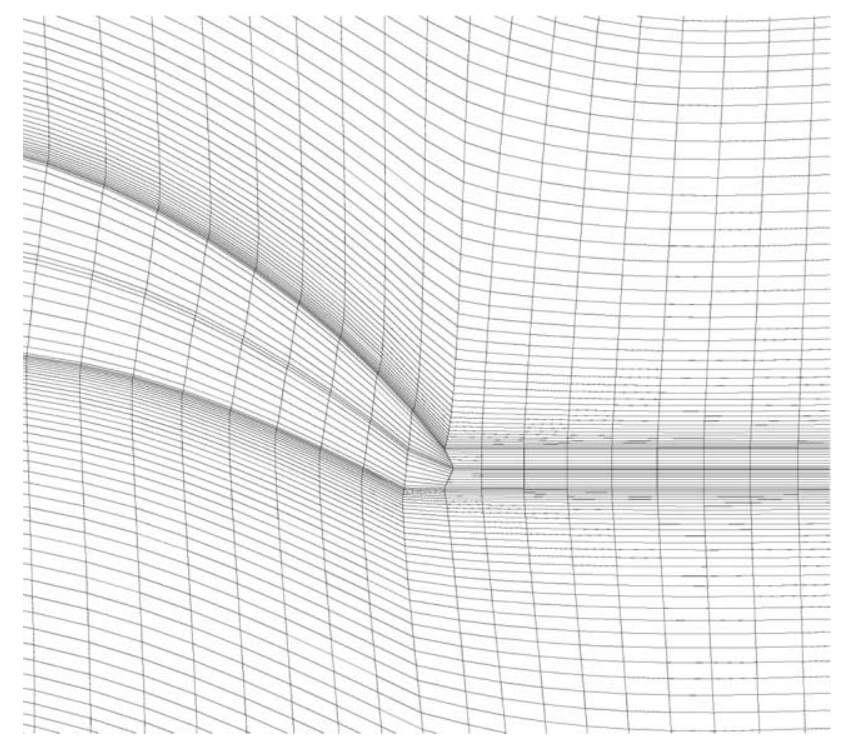

Fig. 10. Example of an embedded $H$-mesh near the blade trailing-edge.

ficulties with non-dissipative numerical schemes. The extremely small $y$ grid spacing in these regions imposes severe restrictions on the allowable time-step size. In addition, the four surface points where the longitudinal and transverse grid lines intercept require special treatment, hence increasing the algorithmic complexity.

The Cartesian mesh IB method is not appropriate for this flow. The rotor blade is quite slender, and thus would be suitable for IB treatment if one set of grid lines could be arranged parallel to the chord. Such an arrangement, however, would make it difficult to impose the appropriate boundary conditions in the $y$-direction, since the flow is not periodic in the direction normal to the chord. Rather, it is periodic in the direction dictated by the blade stagger angle. If the Cartesian mesh is defined with one set of grid lines in the direction along the stagger angle, highly dense resolution will be needed in most of the computational domain in order to resolve the boundary layers on the blade surface, resulting in a large number of grid points.

To overcome the disadvantages of the above methods, the use of IB method on a curvilinear coordinate mesh offers an attractive solution. The emphasis here is 


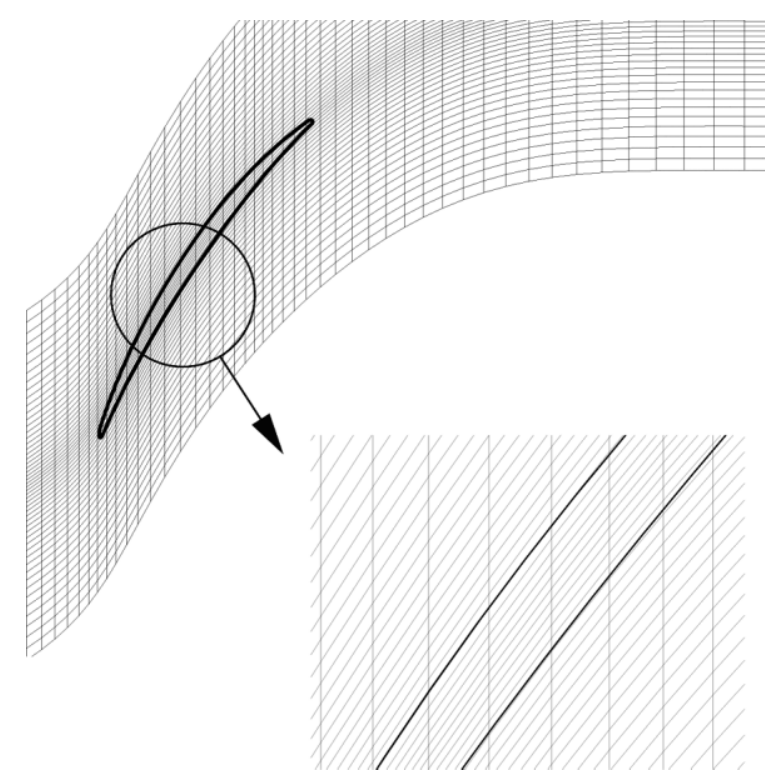

Fig. 11. Curvilinear mesh used in conjunction with the IB method for tip clearance flow (1/4 lines plotted).

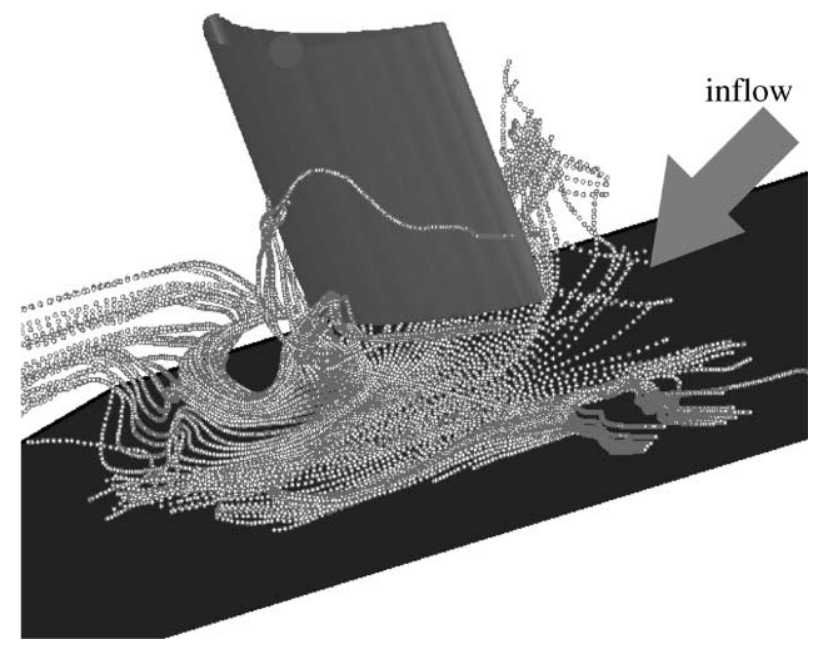

Fig. 12. Streamtraces in the tip-clearance region obtained using the IB method on a curvilinear mesh.

not to save computational cost as in the Cartesian mesh cases discussed in the previous section, but rather, to devise an accurate and flexible treatment of boundary conditions in LES of the tip-clearance flow.

As demonstrated in Fig. 11, the blade surface is nearly parallel to one set of the grid lines, allowing an adequate resolution of the boundary layers. No slip boundary conditions are used on the walls. Periodic boundary conditions can be applied on the (curved) upper and lower boundaries. Preliminary simulations of the 3D tip-clearance flow show satisfactory performance of the method in terms of resolution and numerical stability. LES of the flow shown in Fig. 9 is currently underway at CTR (see Fig. 12).

\section{LES on unstructured grids}

The US Department of Energy's ASCI program has led to an ambitious effort at Stanford to perform an integrated simulation of a gas-turbine engine. The compressor and turbine are simulated using RANS while LES is used for the combustor. This includes the diffuser surrounding the combustion chamber, the injectors, swirlers, dilution holes, etc., which constitute a geometrically very complex configuration (Fig. 16).

The effort spent on grid-generation can be very significant in configurations of this kind; unstructured grids are very desirable in this respect, since the time required for generating unstructured grids is significantly less than that for block-structured grids. However, the bulk of CFD experience with unstructured grids has been in the context of RANS. As pointed out in the introduction, RANS typically uses upwinded numerical methods; although upwinding provides numerical dissipation which makes the solution-procedure robust, when used for LES, this robustness severely compromises accuracy. One solution to this problem is to develop non-dissipative numerical schemes that discretely conserve not only first order quantities such as momentum, but also second-order quantities such as kinetic energy. Discrete conservation of kinetic energy ensures robustness without numerical dissipation. The Harlow and Welch algorithm (1965) possesses this property on structured grids, and has therefore been widely used for LES in simple geometries (see also Morinishi et al., 1998).

Mahesh et al. (2001) have developed a non-dissipative algorithm for turbulent flows computed on unstructured grids. A novel feature of their approach is that it discretely conserves kinetic energy, making it both robust and accurate. A predictor-corrector approach is used to advance the momentum, continuity and the scalar equations.

Discrete energy conservation ensures that the sum:

$\sum_{\text {cvs }} v_{\mathrm{n}} \overrightarrow{\boldsymbol{\Psi}}$

has only contributions from the boundary faces; here cvs refers to the grid volumes and $\overrightarrow{\boldsymbol{\Psi}}$ is the non-linear term in the Navier-Stokes equations. The form of the convection term is known to affect non-linear stability of the discrete equations. The rotational form; i.e., $\vec{u} \times \vec{\omega}-\nabla q^{2}$, and the skew-symmetric form, $\left[\left(u_{i} u_{j}\right)_{, j}+\right.$ $\left.u_{j} u_{i, j}\right] / 2$ have been quite popular for this reason. On tetrahedral or triangular grids, staggered storage of variables allows an elegant implementation of the rotational formulation. The face-normal velocities determine the vorticity component along the edges of the tetrahedra (in 3D), and nodes in (2D). This allows the circulation theorem to be imposed as a constraint on the algorithm (Mahesh et al., 2000). 
While tetrahedral elements allow complex geometries to be easily meshed, they are not the most preferable computational elements for turbulence simulations. Hexahedral elements are preferable-fewer hexahedra fill up a volume; hexahedral elements also generally yield more accurate solutions. The grid may therefore be a combination of arbitrary computational elements; it is partitioned, and then reordered to allow for data locality on each processor and efficient message-passing between processors. Fig. 13 shows scaling data from a run that used up to 1000 processors on ASCI Red-a cluster of 9000 Intel Xeons. Based on the observed speed-up, a five million nodes grid is estimated to use a thousand processors effectively, suggesting that parallel performance is quite satisfactory. The algorithm has been implemented for parallel platforms, and has been tested for a variety of canonical incompressible flows (Mahesh et al., 2000). The robustness of the algorithm is illustrated in Figs. 14 and 15 where even in the inviscid limit, or at very high Reynolds numbers where the dissipative scales are not resolved, the numerical solution mimics the analytical behavior. In contrast a non-dissipative scheme that does not conserve kinetic energy is seen to blow up after some time at high Reynolds numbers. Note that these are very coarse DNS computations and are intended to only demonstrate robustness (not accuracy).

This scheme is being used for LES of a gas turbine combustor. An instantaneous contour plot of the velocity magnitude in the mid-plane of a combustor sector is shown in Fig. 17. No slip boundary conditions are imposed. To demonstrate the importance of accurately accounting for Reynolds number effects (and hence removal of numerical dissipation) the instantaneous contours of velocity magnitude from a simulation at a significantly lower Reynolds number are shown in Fig. 18. The flows at the two Reynolds numbers are qualitatively very different. In the high Reynolds number case the effect of swirl is evident in the outward expansion of

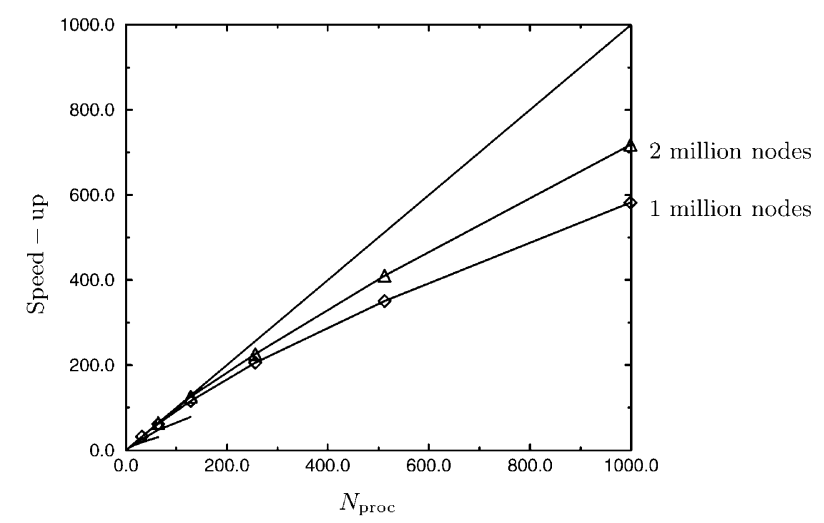

Fig. 13. Results of a scaling study on the ASCI Red computer. The two curves at the bottom left of the figure correspond to grids of 64,000 and 216,000 nodes respectively.

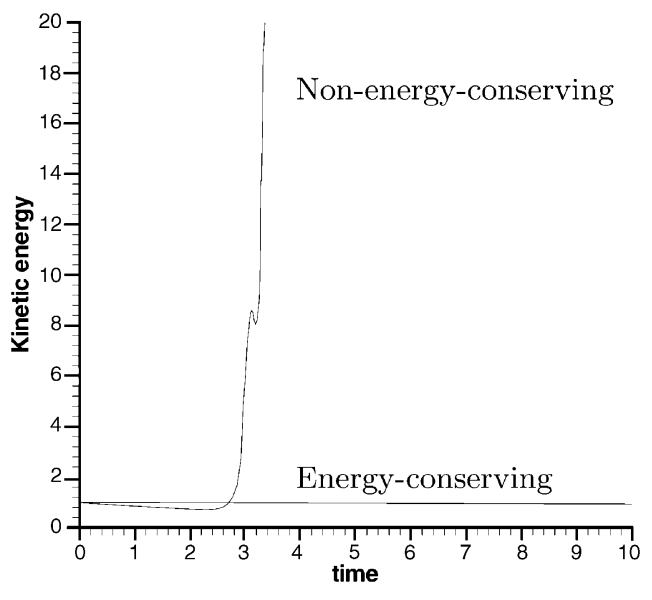

Fig. 14. Illustration of the importance of discretely conserving kinetic energy. The kinetic energy is plotted against time for the Taylor problem at $R e=10^{9}$. The energy-conserving scheme is robust while a non-dissipative scheme that only conserves momentum blows up after some time.

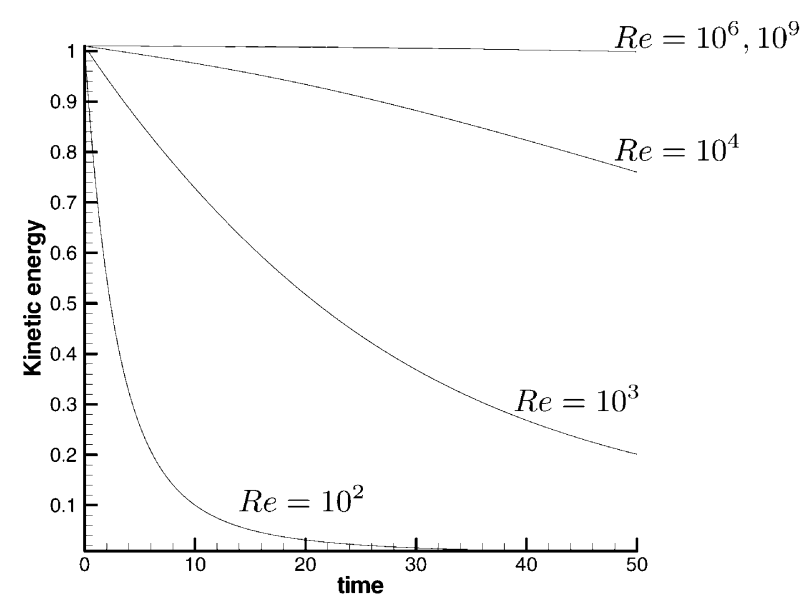

Fig. 15. Kinetic energy in isotropic turbulence is plotted against time at varying Reynolds numbers. Note that the (unstructured mesh) scheme is robust even at the highest Reynolds numbers.

the shear layers and enhanced mixing, whereas in the low Reynolds number flow the flow from the injector is jet like without significant mixing.

\section{Conclusions}

In the past ten years significant progress has been made in the LES technology including development of commuting filters, subgrid scale models and numerical methods for complex configurations. LES has also shown great promise in application to turbulent combustion. Application of LES to industrial problems requires accurate and robust numerical algorithms for complex geometry. Validation in complex flows would also motivate further research and developments of 


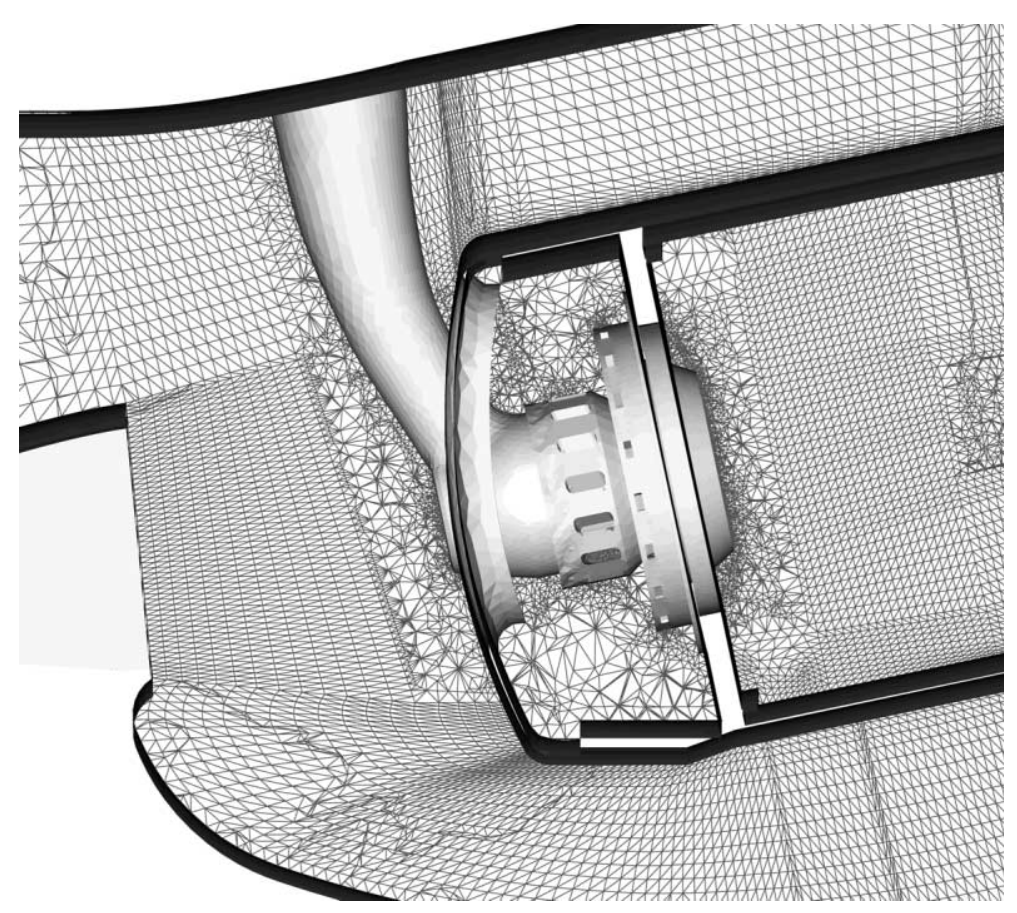

Fig. 16. A cross-section of the combustor geometry and the computational grid.
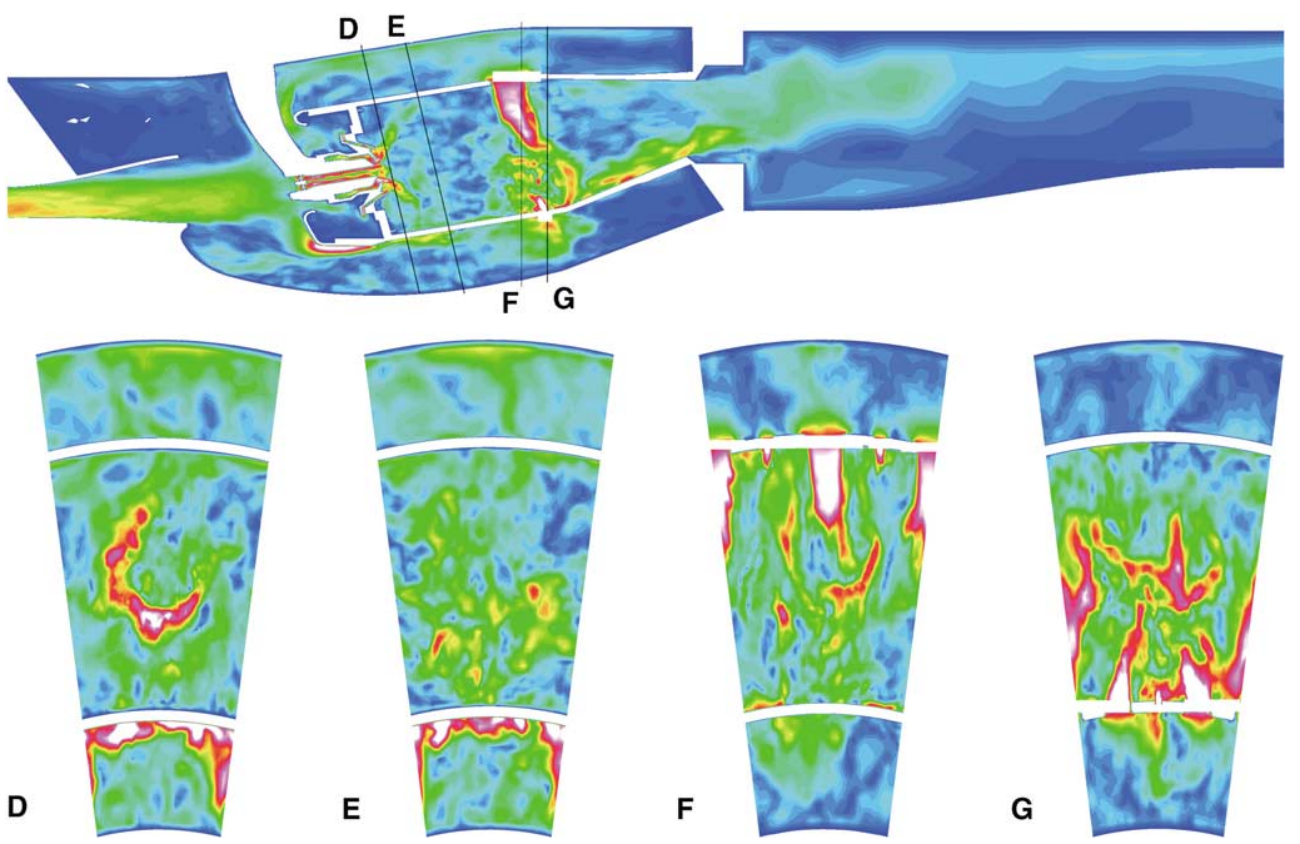

Fig. 17. Contours of instantaneous velocity magnitude in LES of flow in the P\&W combustor geometry. The cross-sectional views in the second row are obtained from the locations indicated as D, E, F and $G$ in the top figure.

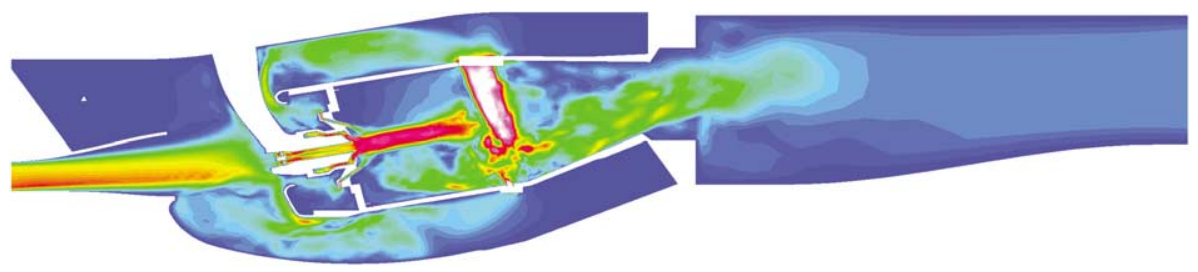

Fig. 18. Contours of instantaneous velocity magnitude in LES of flow in the P\&W combustor geometry at low inlet Reynolds number. 
subgrid scale models which have been tested extensively only in canonical flows. Two numerical methods for LES in complex configurations were presented: a conservative unstructured mesh method used for simulation of flow and combustion in a gas turbine combustor, and the IB method used for flow computations in a stirred tank mixer, over a road vehicle and the tip clearance flow in a stor/rotor combination.

\section{Acknowledgements}

I am grateful to G. Iaccarino, M. Wang, K. Mahesh and $\mathrm{D}$. You for their assistance in the preparation of this manuscript. CTR's work in LES is supported by NASA, US Office of Naval Research, Air Force Office of Scientific Research and the US Department of Energy.

\section{References}

Balaras, E., Benocci, C., Piomelli, U., 1996. Two-layer approximate boundary conditions for large-eddy simulation. AIAA J. 34, 11111119.

Domaradzki, J.A., Loh, K.-C., 1999. The subgrid-scale estimation model in the physical space representation. Physics of Fluids 11, 2330-2342.

Dong, L., Johansen, S.T., Engh, T.A., 1994. Flow induced by an impeller in an unbaffled tank-I. Experimental. Chem. Engng. Sci. 49 (4), 549

Fadlun, E.A., Verzicco, R., Orlandi, P., Mohd-Yusof, J., 2000. Combined immersed-boundary/finite-difference methods for threedimensional complex flow simulations. J. Comp. Phys. 161, 35.

Germano, M., Piomelli, U., Moin, P., Cabot, W.H., 1991. A dynamic subgrid-scale eddy viscosity model. Phys. Fluids A 3, 1760-1765.

Ghosal, S., Lund, T., Moin, P., Akselvoll, K., 1995. A dynamic localization model for large eddy simulation of turbulent flows. J. Fluid. Mech. 286, 229-255.

Gullbrand, J., 2001. Large eddy simulation using explicit filtering in turbulent channel flow. In: Second International Symposium on Turbulence and Shear Flow Phenomena, vol. 2, pp. 359-364.

Harlow, F.H., Welch, J.E., 1965. Numerical calculation of timedependent viscous incompressible flow of fluid with free surface. Phys. Fluids 8, 2182.

Hughes, T.J.R., Mazzei, L., Oberai, A.A., Wray, A.A., 2001. The multiscale formulation of large eddy simulation: decay of homogeneous isotropic turbulence. Phys. Fluids 13, 505-512.

Khalighi, B., Zhang, S., Koromilas, C., Balkanyi, S.R., Bernal, L.P., Iaccarino, G., Moin, P., 2001. Experimental and computational study of unsteady wake flow behind a bluff body with a drag reduction device. SAE Paper, 2001-01B-207.

Kunz, R.F., Lakshminarayana, B., Basson, A.H., 1993. Investigation of tip clearance phenomena in an axial compressor cascade using Euler and Navier-Stokes procedures. J. Turbomach. 115, 453-467.

Mahesh, K., Constantinescu, G., Apte, S., Iaccarino, G., Moin, P., 2001. Large-eddy simulation of gas-turbine combustors, Annual
Research Briefs-2001, Center for Turbulence Research, Stanford University.

Mahesh, K., Constantinescu, G., Moin, P., 2000. Large-eddy simulation of gas-turbine combustors, Annual Research Briefs-2000, Center for Turbulence Research, Stanford University.

Marsden, A.L., Vasilyev, O.V., Moin, P., 2002. Construction of commutative filters for LES on unstructured meshes. J. Comp. Phys. 175, 584-603.

Mittal, R., Moin, P., 1997. Suitability of upwind-biased finite difference schemes for large-eddy simulation of turbulent flows. AIAA J. 35, 1415-1417.

Moin, P., Squires, K., Cabot, W., Lee, S., 1991. A dynamic sub-grid scale model for compressible turbulence and scalar transport. Phys. Fluids A 3, 2746-2757.

Morinishi, Y., Lund, T.S., Vasilyev, O.V., Moin, P., 1998. Fully conservative higher order finite difference schemes for incompressible flow. J. Comput. Phys. 143, 90-124.

Nicoud, F., Baggett, J.S., Moin, P., Cabot, W., 2001. Large eddy simulation wall modeling based on sub optimal control theory and linear stochastic estimation. Phys. Fluids 13, 2968-2984.

O'Rourke, J., 1998. Computational Geometry in C. Cambridge University Press.

Pitsch, H., Steiner, H., 2000. Large eddy simualtion of a turbulent piloted methane/air diffusion flame (Sandia flame D). Phys. Fluids 12, 2541-2554.

Pierce, C., Moin, P., 2001. Progress variable approach for large eddy simulation of turbulent combustion, Report No. TF-80, Flow Physics and Computation Division, Department of Mechanical Engineering, Stanford University.

Piomelli, U., Moin, P., Ferziger, J.H., 1988. Model consistency in large eddy simulation of turbulent channel flows. Phys. Fluids 31, 1884 1891.

Stolz, S., Adams, N.A., Kleiser, L., 2001. An approximate deconvolution model for large eddy simulation with application to incompressible wall-bounded flows. Phys. Fluids 13, 997-1015.

Vasilyev, O.V., Lund, T.S., Moin, P., 1998. A general class of commutative filters for LES in complex geometries. J. Comput. Phys. 146, 82-104.

Vervisch, L., Poinsot, T., 1998. Direct numerical simulation of nonpremixed turbulent flames. Ann. Rev. Fluid Mech. 30, 655691.

Verzicco, R., Fatica, M., Iaccarino, G., Moin, P., Khalighi, B., 2001. Large Eddy Simulation of a Road-Vehicle with Drag Reduction Devices, CTR Manuscript 179, Center For Turbulence Research, AIAA J., submitted for publication.

Verzicco, R., Iaccarino, G., Fatica, M., Orlandi, P., 2000a. Flow in an impeller stirred tank using an immersed boundary technique. Annual Research Briefs 2000, Center For Turbulence Research, p. 251. AIChE J., submitted for publication.

Verzicco, R., Mohod-Yusof, J., Orlandi, P., Haworth, D., 2000b. Large-eddy-simulation in complex geometric configurations using boundary body forces. AIAA J. 38 (3), 427.

You, D., Mittal, R., Wang, M., Moin, P., 2002. Large eddy simulation of a rotor tip-clearance flow. AIAA Paper 2002-0981.

Wang, M., Moin, P., 2001. Wall modeling in LES of trailing-edge flow. In: Second International Symposium on Turbulence and Shear Flow Phenomena, vol. 2, pp. 165-170.

Wang, Y., 2000. Tip leakage flow downstream of a compressor cascade with moving end wall, Master Thesis, Department of Aerospace Engineering, Virginia Polytechnic Institute and State University. 